\title{
Skin Reduction Nipple-Sparing Mastectomy
}

\section{Jill Dietz ${ }^{1}$ and Greg Fedele ${ }^{2}$}

${ }^{1}$ Case Western Reserve School of Medicine, Seidman Cancer Center, Cleveland, OH; ${ }^{2}$ Center for Plastic and Cosmetic Surgery, Cleveland, $\mathrm{OH}$

\begin{abstract}
Nipple-sparing mastectomy has been shown to be a safe and effective treatment for many patients with early stage breast cancer. Large-breasted women who are gene positive or who have early breast cancer are not traditionally candidates for nipple-sparing mastectomy. The blood supply to the nipple is via the breast tissue and also the dermis, both of which would be severed if standard skin reduction were combined with mastectomy. This video shows how a nipple-sparing mastectomy can be combined with skin reduction while preserving the blood supply to
\end{abstract}

the nipple. The excess skin is deepithelialized to preserve the dermal vessels. After mastectomy, the excess skin is imbricated to reduce the skin envelope. The deepithelialized lower flap "autoderm" can be sewn to the pectoralis muscle for coverage of the tissue expander. This technique allows large-breasted and ptotic patients to undergo mastectomy with preservation of their nipple-areolar complex as well as skin reduction to yield an improved cosmetic result.
Electronic supplementary material The online version of this article (doi:10.1245/s10434-015-4722-x) contains supplementary material, which is available to authorized users.

(C) Society of Surgical Oncology 2015

First Received: 14 April 2015;

Published Online: 13 August 2015

J. Dietz

e-mail: jill.dietz@uhhospitals.org 